\title{
Introduction to the Minitrack on Interactive Visual Analytics and Visualization for Decision Making
}

\author{
David S. Ebert \\ Purdue University \\ ebertd@purdue.edu
}

\author{
Brian Fisher \\ Simon Fraser University \\ bfisher@sfu.ca
}

\author{
Kelly Gaither \\ University of Texas \\ kelly@tacc.utexas.edu
}

The topic of this minitrack, Interactive Visual Analytics and Visualization for Decision Making, has applications in a broad range of situations where human expertise must be brought to bear on problems characterized by massive datasets and data that are uncertain in fact, relevance, location in space and position in time. Examples include environmental science and technologies, natural resources and energy, health and related life sciences, precision medicine, safety and security (aircraft safety, law enforcement, antiterrorism, disaster relief) and business processes. Key research challenges of interest in this area include studies of visual analytics and decision support, interactive performance related to serving up information with a short time of relevance, and collaborative analysis using visual information systems. Interactive Visual Analytics and Visualization for Decision Making supports human decision making through interaction with data and statistical and machine learning processes, with applications in a broad range of situations where human expertise must be brought to bear on problems characterized by massive datasets and data that are uncertain in fact, relevance, location in space and position in time. Current applications include environmental science and technologies, natural resources and energy, health and related life sciences, precision medicine, safety and security and business processes.

The focus in this minitrack goes beyond analytics to include rich, powerful visualization techniques for turning data into actionable information. These rich, interactive visual analytic environments offer even greater power and promise to solve big data problems for data that is "big" in any of the dimensions of variability, velocity, or volume. This minitrack builds upon earlier HICSS minitracks on visual analytics, mobile computing, and digital media at scale, focusing more decision analytics in various applications from business to science, natural disasters, public safety, and policy.

One paper selected for this minitrack, "A Visual Analytics Approach for Multi-scale Resume Analysis," presents a visual analytics system ResumeVis -- a text-mining approach presented to extract semantic information. A set of visualizations are devised to represent resumes in multiple URI: http://hdl.handle.net/10125/50046 ISBN: 978-0-9981331-1-9

(CC BY-NC-ND 4.0) perspectives. By interactive exploration on ResumeVis, three tasks can be accomplished: to trace individual career trajectories; to mine latent socialrelations among individuals; and to capture collective mobility in massive resumes. Case studies with 2,640 officer resumes demonstrate the effectiveness. The paper presents a visual analytics prototype based on resume data, aiming to help users understand the semantic patterns hidden in the resumes.

A second paper, "Immersive Visualization for Abnormal Detection in Heterogeneous Data for Onsite Decision Making," presents an immersive visualization approach for investigating abnormal events in heterogeneous, multi-source, and timeseries sensor data collections in real-time on the site of the event. This approach explores the essential components for an analyst to visualize complex data and explore hidden connections in mixed reality and combines automatic event detection algorithms to identify suspicious activities.

A third paper, "Representation Effects and Loss Aversion in Analytical Behaviour: An Experimental Study into Decision Making Facilitated by Visual Analytics," investigate the relationship between the representation of data and decision making. Three hundred participants were asked to decide between four pairs of simulated stock market investment choices. Each pair varies in the amount of risk and reward and all stocks are presented as line charts. Participants were divided into three equal sized groups and shown different representations of the same data to test the representation effect; variations in aspect ratios and the use of dual-line charts over two single-line charts. The results reveal a consistent main effect and two significant interactions between visualisations and decision making.

These papers show a wide range of visualization and analytics in complex decision making environments and provide valuable insights into the design, production, and deployment of visual analytics applicable to most decision and discovery tasks across a broad spectrum of applications. Moreover, they clearly demonstrate effective ways to harness and tame big data for discovery, insight, management, and action.

\section{$\mathrm{HI}$ C S S}


Looking back over the evolution of visual analytics for decision making, as shown in past mini-tracks, it is clear that the uses of visual decision analytics has grown and spread to more fields and we see this trend continuing at an accelerated rate. As more data becomes available in every aspect of decision making, the role and importance of interactive visual decision analytics will become critical in effective and efficient decision making. We hope you will join us for interesting presentations and lively discussions on new visual analytics techniques and solutions for our evolving landscape of problems requiring rapid and reliable decision-making. 